\title{
Discrimination between human populations using a small number of differentially methylated CpG sites: a preliminary study using lymphoblastoid cell lines and peripheral blood samples of European and Chinese origin
}

\author{
Patrycja Daca-Roszak ${ }^{*}$ (D), Roman Jaksik², Julia Paczkowska', Michał Witt ${ }^{1}$ and Ewa Ziętkiewicz
}

\begin{abstract}
Background: Epigenetics is one of the factors shaping natural variability observed among human populations. A small proportion of heritable inter-population differences are observed in the context of both the genome-wide methylation level and the methylation status of individual CpG sites. It has been demonstrated that a limited number of carefully selected differentially methylated sites may allow discrimination between main human populations. However, most of the few published results have been performed exclusively on B-lymphocyte cell lines.

Results: The goal of our study was to identify a set of $\mathrm{CpG}$ sites sufficient to discriminate between populations of European and Chinese ancestry based on the difference in the DNA methylation profile not only in cell lines but also in primary cell samples. The preliminary selection of $\mathrm{CpG}$ sites differentially methylated in these two populations (pop-CpGs) was based on the analysis of two groups of commercially available ethnically-specific Blymphocyte cell lines, performed using Illumina Infinium Human Methylation 450 BeadChip Array. A subset of 10 pop-CpGs characterized by the best differentiating criteria (|Mdiff $\mid>1, q<0.05$; lack of the confounding genomic features), and 10 additional CpGs in their immediate vicinity, were further tested using pyrosequencing technology in both B-lymphocyte cell lines and in the primary samples of the peripheral blood representing two analyzed populations. To assess the population-discriminating potential of the selected set of CpGs (further referred to as "composite pop (CEU-CHB)-CpG marker"), three classification methods were applied. The predictive ability of the composite 8-site pop (CEU-CHB)-CpG marker was assessed using 10-fold cross-validation method on two independent sets of samples.
\end{abstract}

(Continued on next page)

* Correspondence: patrycja.daca-roszak@igcz.poznan.pl

${ }^{1}$ Institute of Human Genetics, Polish Academy of Sciences, Strzeszynska 32, 60-479 Poznan, Poland

Full list of author information is available at the end of the article

(c) The Author(s). 2020 Open Access This article is licensed under a Creative Commons Attribution 4.0 International License, which permits use, sharing, adaptation, distribution and reproduction in any medium or format, as long as you give appropriate credit to the original author(s) and the source, provide a link to the Creative Commons licence, and indicate if changes were made. The images or other third party material in this article are included in the article's Creative Commons licence, unless indicated otherwise in a credit line to the material. If material is not included in the article's Creative Commons licence and your intended use is not permitted by statutory regulation or exceeds the permitted use, you will need to obtain permission directly from the copyright holder. To view a copy of this licence, visit http://creativecommons.org/licenses/by/4.0/. The Creative Commons Public Domain Dedication waiver (http://creativecommons.org/publicdomain/zero/1.0/) applies to the data made available in this article, unless otherwise stated in a credit line to the data. 
(Continued from previous page)

Conclusions: Our results showed that less than 10 pop-CpG sites may distinguish populations of European and Chinese ancestry; importantly, this small composite pop-CpG marker performs well in both lymphoblastoid cell lines and in non-homogenous blood samples regardless of a gender.

Keywords: DNA methylation, Human population identification, Pyrosequencing, Population differentiating CpGs

\section{Background}

Genetic variation of human populations is extensively explored in a variety of fields including epidemiological and medical studies (e.g. population-specific susceptibility to diseases, pharmacogenomics), but also in evolutionary studies and forensics (e.g. populations origin, relationships, identification) [1-5]. The relation between the genome variation and population ancestry has been admittedly proven [6-9]. A variety of genomic markers (SNPs, CNVs, microsatellites, and mtDNA, Y-chromosome haplotypes) providing accurate ancestry information have been identified, validated and successfully implanted in populationstratification tests (e.g. [10-12]).

The differences between human populations are shaped not only by the genomic DNA variation but also by transcriptomic and DNA methylation variation [1322]. Therefore, besides the most frequently used genomic DNA markers, some "non-classical markers", representing inter-population differences in the expression and in the DNA methylation level, can potentially be used to discriminate between populations. In fact, a number of population-specific mRNA markers have been identified and tested in both B-cell lines and in a primary biological material, e.g. blood see [23].

It is well known that the majority of differences in the level of DNA methylation are caused by multiple environmental factors e.g. nutrition, exposure to pollutants, social conditions, etc. [24-27]. However, the recent development of high-throughput methods (mainly microarray technology) provided a wealth of data, which have demonstrated that a considerable part of the methylation variance reflects stable and heritable differences [28, 29]. Some of them are inter-individual and some differentiate populations [13, 18-20, 30-32]. The inter-population differences are observed in both the genome-wide methylation level and in the methylation status of individual CpG sites [15, 16, 19, 20, 33-35]. Compared to the genomic DNA variation, the persistent interpopulation differences in the methylation level are rather small; nevertheless, they represent a possible source of markers that could be used for human population stratification. The inter-population differences in the level of methylation have been demonstrated in distinct types of a biological material: B-lymphocyte cell lines (e.g. [19, 20, 36, 37]), skin cells (e.g. [38, 39]), blood samples (e.g. $[13,30])$. Moreover, it has been shown that even a limited number ( $400 \mathrm{CpGs})$ of carefully selected differentially methylated $\mathrm{CpG}$ sites may allow discrimination of three main human groups: Americans of African origin, Europeans and Asians [20].

The goal of our study was to identify a small set of differentially methylated CpG sites (pop-CpGs) sufficient to discriminate between populations of European and Chinese ancestry, which could be used as an easily manageable, composite pop (CEU-CHB)-CpG marker for a forensic differentiation between samples based on their population origin (see Fig. 1).

A set of $14 \mathrm{CpG}$ sites characterized by significant population differences in their methylation ( $\mid$ Mdiff $\mid>1$ at $\mathrm{q}<0.05$, and the lack of confounding SNPs under Illumina probes) was identified, based on the analysis of 36 commercially available B-lymphocyte cell lines of European and Chinese origin, performed using Illumina Infinium Human Methylation 450 BeadChip Array. A subset of $10 \mathrm{CpGs}$ characterized by the best criteria, and 10 additional CpGs in their immediate vicinity, was further tested in both B-lymphocyte cell lines and in primary samples of peripheral blood. Statistical evaluation of the discriminating potential of the best-performing pop-CpGs, employing 10-fold cross-validation method, was then performed in two independent sets of samples.

\section{Results}

Selection of candidate pop-CpGs

Illumina Infinium HumanMethylation 450 BeadChip

Array (HM450K array), previously applied to characterize methylation level in B-lymphocyte cell lines representing $\mathrm{CEU}(n=18)$ and $\mathrm{CHB}(n=18)$, revealed a set of $96 \mathrm{CpGs}$, differentiating the two populations at the significance level $p<0.05$, and representing the highest inter-population differences in the average methylation levels $\left(\mid \mathrm{M}_{\mathrm{av} \_}\right.$diff $\left.\mid>1 ; \mathrm{q}<0.05\right)$ see [40]. From these differentially methylated CpGs, a small set of 14, characterized by the absence of confounding features (lack of SNPs in the studied CpG, lack of frequent SNPs under Illumina probe; no multi-site mapping of the probe), was selected as candidate pop-CpGs (Table 1).

Eleven of 14 best-differentiating CpGs were located outside CpG islands (in shore or shelf regions, gene body, transcription site start or 5'UTR regions). Three CpG sites, cg04036182 (chr15:45458818), cg07207043 (chr6:7051497) and cg00031303 (chr3: 195681400), were 


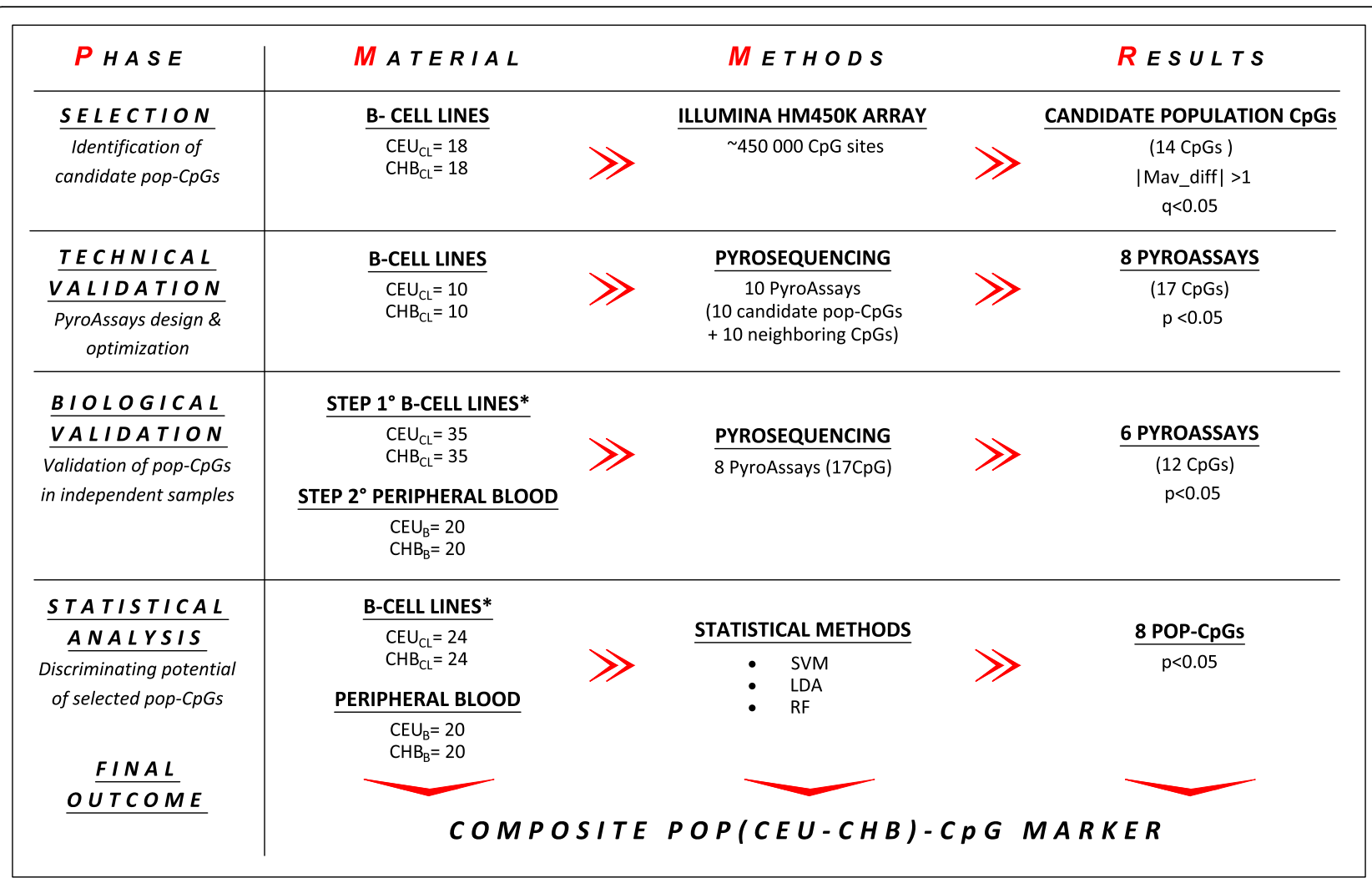

Fig. 1 Study design. * cell lines other than those used in Illumina study. Authors' original figure

located in the genomic island of SHF, RREB1 and SDHA P1 genes, respectively. The highest inter-population differences in the methylation level ( $\sim 40 \%$ difference) were observed in cg18136963 (chr6:139013146) and cg26367031 (chr3:178984747) ( $\mathrm{M}_{\mathrm{av} \_}$diff $\left.\geq 2.7\right)$.
DNA methylation and gene expression correlation analysis

Thirty-six B-lymphocyte cell lines from both populations (CEU and $\mathrm{CHB}$ ) were analyzed on HM450 array (Illumina) and HumanHT-12v4 Expression BeadChip Kit expression array (Illumina). Based on the results obtained

Table 1 Characteristics of the candidate pop-CpGs

\begin{tabular}{|c|c|c|c|c|c|c|c|}
\hline $\mathrm{nb}$ & Candidate pop- CpGs & Genomic position (GRCh:37) & Locus & Gene region & Type of region & $\mid M_{\text {av__ }}$ diff $\mid$ & q-value \\
\hline 1 & cg18136963 & chr6:139013146 & FLJ49 & not provided & N_Shore & 2.950 & 0.0355 \\
\hline 2 & cg26367031 & chr3:178984747 & KCNMB3 & 5'UTR; 1st exon & not provided & 2.775 & 0.0215 \\
\hline 3 & cg03140118 & chr1:37939320 & ZC3H12A & TSS1500 & N_Shore & 2.411 & 0.001 \\
\hline 4 & cg23669876 & chr1: 36489276 & AGO3 & Body (LTR) & not provided & 2.355 & 0.0039 \\
\hline 5 & cg00862290 & chr3:178984973 & KCNMB3 & TSS200 & S_Shore & 2.247 & 0.008 \\
\hline 6 & cg08979191 & chr5:132113734 & SEPT8 & TSS200 & S_Shore & 1.875 & 0.0185 \\
\hline 7 & $\operatorname{cg} 24037715$ & chr14: 35203968 & - & not provided & sea & 1.691 & 0.0003 \\
\hline 8 & cg07207043 & chr6:7051497 & RREB1 & not provided & CpG Island & 1.534 & 0.0345 \\
\hline 9 & cg04036182 & chr15:45458818 & SHF & not provided & CpG Island & 1.451 & 0.0201 \\
\hline 10 & cg00031303 & chr3: 195681400 & SDHAP1 & not provided & CpG Island & 1.359 & 0.005 \\
\hline 11 & cg07904028 & chr4:6328508 & PPP2R2C & body & not provided & 1.257 & 0.0145 \\
\hline 12 & cg09972454 & chr16: 15083088 & PDXDC1 & body & N_Shore & 1.232 & 0.0029 \\
\hline 13 & cg24861686 & chr8:11418058 & $B L K$ & body & N_Shelf & 1.193 & 0.000 \\
\hline 14 & cg03585734 & chr1: 15598865 & FHAD1 & body & not provided & 1.123 & 0.0144 \\
\hline
\end{tabular}

CpGs selected for pyrosequencing validation are bolded. Shores and shelves are defined in Illumina as regions 0-2 kb and 2-4 kb, respectively, from a CpG island. $N$ Upstream, S Downstream, TSS Transcription site start, LTR Long terminal region 
from both Illumina platforms, a t-test was performed to identify CpG loci and genes, showing statistically significant inter-population differences in the level of DNA methylation and in the gene expression, respectively. Subsequently, to identify a relation between the gene expression and the corresponding methylation status, a Pearson correlation analysis was performed.

Based on the two-step statistical analysis, a group of genes and CpG loci meeting statistical criteria, $p<$ 0.01 in t-tests and in Pearson correlation analysis, was identified. None of the pop-CpGs, except for cg24861686 (1_CpG1, chr8:11418058), met the abovementioned statistical criteria. This CpG site showed positive correlation with $B L K$ gene (Pearson coefficient 0.63).

\section{Technical validation}

A subset of 10 pop-CpGs candidates meeting even more stringent statistical criteria $\left(\mid \mathrm{M}_{\mathrm{av}-}\right.$ diff $\mid \geq 1.2$ at $\left.\mathrm{q}<0.05\right)$, and 10 additional CpGs located in their close proximity, was analyzed using pyrosequencing technique (Table 2).
Due to technical reason (see Additional file 1 for details), some CpGs were excluded, and a subset of 17 CpGs was analyzed in further experiments.

Pyrosequencing results were collected as proportional values, separately for each analyzed CpG site (Table 2, Fig. 2). The average value of differences in methylation level between the studied populations was in the range of 0.119 (PyroAssay 6_CpG1 chr15:45458826) to 0.387 (PyroAssay 2_CpG1 chr1:37939320). Statistically significant population differences $(p<0.05)$ were obtained for most of the CpG sites. The results from pyrosequencing were concordant with the results from HM450K array. The only exception was PyroAssay 5, where no statistically significant population differences in the level of methylation were noted for two out of the three examined CpGs (5_CpG2 chr5:132113755 and 5_CpG3 chr5: 132113777); nevertheless, this PyroAssay was not excluded from further analyzes.

Figure 2 shows the distribution of methylation levels in individual B-lymphocyte cell lines used in the technical validation phase. Eight PyroAssays $(1,2,3,5,6$, 8 ,

Table 2 Comparison of DNA methylation levels assessed using Illumina HM450K array and pyrosequencing assays (PyroAssays)

\begin{tabular}{|c|c|c|c|c|c|c|c|c|c|}
\hline \multirow{2}{*}{$\begin{array}{l}\text { CpG name } \\
\text { in HM450K } \\
\text { array }\end{array}$} & \multirow{2}{*}{$\begin{array}{l}\text { PyroAssay } \\
\text { name }\end{array}$} & \multicolumn{4}{|c|}{ Illumina infinium human methylation 450BEAD chip array } & \multicolumn{4}{|c|}{ Pyrosequencing technical validation } \\
\hline & & $\begin{array}{l}\text { beta_mean_CEU } \\
(n=18)\end{array}$ & $\begin{array}{l}\text { beta_mean_CHB } \\
(n=18)\end{array}$ & $\begin{array}{l}\text { CEU.beta } \\
\text { mean } \\
\text {-CHB.beta_ } \\
\text { mean }\end{array}$ & $\begin{array}{l}\mathrm{q}^{-} \\
\text {value }\end{array}$ & $\begin{array}{l}\text { CEU.mean } \\
(n=10)\end{array}$ & $\begin{array}{l}\text { CHB.mean } \\
(n=10)\end{array}$ & $\begin{array}{l}\text { CEU.mean } \\
\text {-CHB.mean }\end{array}$ & $\begin{array}{l}p \text {-value } \\
\text { beta }\end{array}$ \\
\hline $\operatorname{cg} 24861686$ & 1_CpG1 ${ }^{a}$ & 0.841 & 0.697 & 0.143 & 0.0000 & 0.813 & 0.591 & 0.222 & 0.0000 \\
\hline \multirow[t]{3}{*}{ cg03140118 } & 2_CpG1 $1^{a}$ & 0.176 & 0.503 & -0.327 & 0.0010 & 0.131 & 0.518 & -0.387 & 0.0003 \\
\hline & 3_CpG1 & - & - & - & - & 0.410 & 0.150 & 0.259 & 0.0056 \\
\hline & 3_CpG2 & - & - & - & - & 0.289 & 0.087 & 0.202 & 0.0048 \\
\hline cg00862290 & 3_CpG3 $3^{\mathrm{a}}$ & 0.466 & 0.161 & 0.305 & 0.0080 & - & - & - & - \\
\hline cg07904028 & 4_CpG1 $1^{a}$ & 0.515 & 0.714 & -0.199 & 0.0145 & - & - & - & - \\
\hline \multirow[t]{4}{*}{ cg08979191 } & 5_CpG1 ${ }^{a}$ & 0.779 & 0.520 & 0.258 & 0.0185 & 0.782 & 0.544 & 0.238 & 0.0117 \\
\hline & 5_CpG2 & - & - & - & - & 0.609 & 0.400 & 0.209 & 0.1174 \\
\hline & 5_CpG3 & - & - & - & - & 0.599 & 0.418 & 0.181 & 0.1942 \\
\hline & 6_CpG1 & - & - & - & - & 0.067 & 0.186 & -0.119 & 0.0106 \\
\hline cg04036182 & 6_CpG2 $2^{a}$ & 0.271 & 0.486 & -0.215 & 0.0201 & 0.112 & 0.470 & -0.358 & 0.0000 \\
\hline $\operatorname{cg} 26367031$ & 7_CpG1 ${ }^{a}$ & 0.539 & 0.170 & 0.369 & 0.0215 & - & - & - & - \\
\hline \multirow[t]{3}{*}{ cg18136963 } & 8_CpG1 & - & - & - & - & 0.520 & 0.179 & 0.341 & 0.0019 \\
\hline & 8_CpG2 ${ }^{a}$ & 0.514 & 0.162 & 0.352 & 0.0355 & 0.498 & 0.174 & 0.324 & 0.0097 \\
\hline & 8_CpG3 & - & - & - & - & 0.423 & 0.179 & 0.243 & 0.0180 \\
\hline \multirow[t]{4}{*}{ cg07207043 } & 9_CpG1 ${ }^{a}$ & 0.625 & 0.820 & -0.195 & 0.0345 & 0.529 & 0.813 & -0.283 & 0.0023 \\
\hline & 9_CpG2 & - & - & - & - & 0.422 & 0.726 & -0.304 & 0.0007 \\
\hline & 9_CpG3 & - & - & - & - & 0.480 & 0.814 & -0.335 & 0.0004 \\
\hline & 10_CpG1 & - & - & - & - & 0.258 & 0.531 & -0.272 & 0.0000 \\
\hline cg23669876 & 10_CpG2 ${ }^{\mathrm{a}}$ & 0.368 & 0.728 & -0.360 & & 0.290 & 0.590 & -0.299 & 0.0000 \\
\hline
\end{tabular}




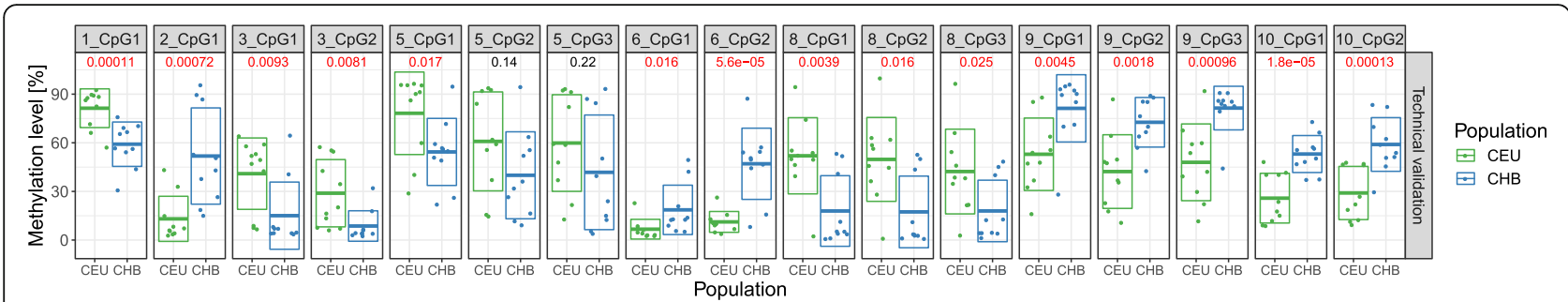

Fig. 2 Results of the technical validation of eight PyroAssays. Twenty B-lymphocyte cell lines (10 from each population) were tested. The originally selected candidate pop-CpGs targeted in each PyroAssay are marked with *. Green - CEU population; blue - CHB population. Dots represent methylation levels in individual samples. Box plots denote mean value (lines inside the boxes) and standard deviation. Statistically significant $(p<0.05)$ population differences in the methylation level are marked in red

9 and 10) passed the technical validation and were used in the further step of biological validation.

\section{Biological validation of population differences in methylation level \\ Independent B-lymphocyte cell lines}

To test the biological validity of population-differentiating methylation status of $17 \mathrm{CpG}$ sites, eight PyroAssays were performed in the independent set of B-lymphocyte cell lines. Statistically significant $(p<0.05)$ population differences in the mean methylation level were observed for 6 out of 8 tested PyroAssays (covering 12 CpG sites, see Table 3).

In the majority of PyroAssays, the level of methylation was similar across the neighboring CpG sites (Table 3). Only two CpGs (5_CpG3 chr5:132113777 and 9_CpG1 chr6:7051497) had distinct methylation level compared to the rest of positions targeted by the respective PyroAssay, with no statistically significant differences between the two populations (Table 3). The highest interpopulation differences in methylation level were noted for CpGs covered by PyroAssays 8 and 10 (Table 3, CEUmean-CHBmean column). PyroAssays 2 and 3 didn't reveal any statistically significant population differences in CpG methylation.

\section{Peripheral blood samples}

To test, whether population differences in the methylation levels of CpGs observed in CEU and CHB cell lines, reflected real differences between the two populations (and were not due to the cell lines' peculiarities), the second step of biological validation was performed, using a primary biological material, i.e. peripheral blood samples

Table 3 Validation of eight PyroAssays performed in the independent set of B-lymphocyte cell lines

\begin{tabular}{|c|c|c|c|c|c|c|c|c|c|}
\hline $\begin{array}{l}\text { PyroAssay number_position } \\
\text { of CpG in the assay }\end{array}$ & CEU (n) & CHB (n) & CEU.mean & CHB.mean & CEU.var & CHB.var & CEU.mean - CHB.mean & padj_beta & $\begin{array}{l}\text { Pop_diff } \\
\text { potential }\end{array}$ \\
\hline 1_CpG1 & 34 & 34 & 0.800 & 0.759 & 0.008 & 0.006 & 0.040 & 0.032 & 1 \\
\hline 2_CpG1 & 34 & 34 & 0.243 & 0.252 & 0.052 & 0.040 & -0.008 & 0.723 & 0 \\
\hline 3_CpG1 & 34 & 34 & 0.246 & 0.222 & 0.069 & 0.051 & 0.024 & 0.828 & 0 \\
\hline 3_CpG2 & 34 & 34 & 0.203 & 0.168 & 0.044 & 0.031 & 0.035 & 0.696 & 0 \\
\hline 5_CpG1 & 34 & 34 & 0.718 & 0.594 & 0.057 & 0.041 & 0.124 & 0.049 & 1 \\
\hline 5_CpG2 & 34 & 34 & 0.561 & 0.420 & 0.046 & 0.046 & 0.141 & 0.040 & 1 \\
\hline 5_CpG3 & 34 & 34 & 0.522 & 0.448 & 0.064 & 0.049 & 0.074 & 0.319 & 0 \\
\hline 6_CpG1 & 34 & 34 & 0.132 & 0.242 & 0.017 & 0.029 & -0.110 & 0.007 & 1 \\
\hline 6_CpG2 & 34 & 34 & 0.236 & 0.343 & 0.036 & 0.031 & -0.107 & 0.018 & 1 \\
\hline 8_CpG1 & 35 & 35 & 0.481 & 0.180 & 0.111 & 0.039 & 0.301 & 0.000 & 1 \\
\hline 8_CpG2 & 35 & 35 & 0.492 & 0.166 & 0.125 & 0.050 & 0.325 & 0.000 & 1 \\
\hline 8_CpG3 & 35 & 35 & 0.459 & 0.193 & 0.108 & 0.050 & 0.267 & 0.002 & 1 \\
\hline 9_CpG1 & 34 & 34 & 0.713 & 0.806 & 0.042 & 0.035 & -0.093 & 0.075 & 0 \\
\hline 9_CpG2 & 34 & 34 & 0.632 & 0.772 & 0.035 & 0.021 & -0.140 & 0.001 & 1 \\
\hline 9_CpG3 & 34 & 34 & 0.657 & 0.784 & 0.049 & 0.030 & -0.127 & 0.017 & 1 \\
\hline 10_CpG1 & 30 & 31 & 0.146 & 0.561 & 0.035 & 0.055 & -0.415 & 0.000 & 1 \\
\hline 10_CpG2 & 30 & 31 & 0.171 & 0.640 & 0.043 & 0.062 & -0.469 & 0.000 & 1 \\
\hline
\end{tabular}

CpG sites characterized by statistically significant inter-population differences in their methylation level are bolded. padj_beta: p-value after Benjamin Hochberg correction; pop-diff potential: differentiation potential of individual sites: 0-non-differentiating; 1-differentiating 
from individuals representing two analyzed populations ( $n=40$ from both CEU and CHB).

Overall, PyroAssays revealed similar inter-population differences in the level of CpG methylation in both Blymphocyte cell lines and in blood samples. Furthermore, similar to the results obtained in B-lymphocyte cell lines, a high consistency in the methylation level among individual CpG sites examined within a given PyroAssay was also observed in blood samples (Fig. 3). The greatest inter-population differences in the level of CpG methylation was observed in PyroAssays 8 and 5 . Only few inconsistencies were observed between Blymphocyte cell lines and blood samples. Population differences in the methylation of 5_CpG3 (chr5:132113777) and 9_CpG1 (chr6:7051497) sites, which did not reach statistical significance in B-cell lines, were statistically significant in blood samples, whereas the interpopulation differences in 1_CpG1 (chr8:11418058) were not significant in blood samples. On the other hand, CpG sites targeted by PyroAssay 10, which classified as strongly population-differentiating sites in the B-cell lines, in blood samples were characterized by the lowest average differences in their methylation values.

For the majority of PyroAssays, methylation readouts in individual blood samples were tightly clustered, as opposed to those observed in B-lymphocyte cell lines. The only exception was PyroAssay 8, where the spread of the readouts from blood samples was much larger, and had a clear a tri-modal methylation distribution (see Discussion).

\section{Discriminating potential of the selected pop-CpGs Identification of a composite pop (CEU-CHB)-CpG marker} Pearson correlation analysis was performed using data from B-lymphocyte cell lines analysis $(n=10 \mathrm{CEU} ; n=$ $10 \mathrm{CHB}$ ) obtained during the technical validation step.
Analysis showed a high correlation coefficient (0.8-1) within each of the corresponding PyroAssays, and simultaneously a low correlation $(<0.5)$ between individual PyroAssays (see Fig. 4 below).

To select the non-redundant set of validated pop-CpGs, correlated sites identified in the Pearson correlation analysis in each of the PyroAssays were removed. Based on the $p$-value after Benjamin Hochberg correction (the lowest padj_beta values were selected, see Table 3), a set of eight CpG sites (1_CpG1 chr8:11418058, 2_CpG1 chr1: 37939320, 3_CpG2 chr3:178984959, 5_CpG1 chr5: 132113734, 6_CpG2 chr15:45458818, 8_CpG1 chr6: 139013142, 9_CpG3 chr6:7051504, 10_CpG1 chr1: 36489272) was selected. This set of eight non-redundant, validated pop-CpGs formed a composite pop (CEU$\mathrm{CHB}$ - $\mathrm{CpG}$ marker, with the potential to discriminate between $\mathrm{CEU}$ and $\mathrm{CHB}$ populations based on the differences in the level of methylation.

\section{Testing of the composite pop (CEU-CHB)-CpG marker}

To assess the population-discriminating potential of the 8-site composite pop (CEU-CHB)-CpG marker, three different classification methods were used: support vector machines (SVM) with linear kernel, linear discriminant analysis (LDA) and random forest (RF). The predictive ability of each method was assessed using 10fold cross-validation, which was repeated 1000 times due to the moderate number of available cases.

The results obtained using each of the classification algorithms (SVM, LDA and RF) were compared in terms of AUC parameter (area under ROC curve) (see Fig. 5).

The shape of all presented curves followed the lefthand corner and the top border, indicating the high accuracy of the 8-site composite pop (CEU-CHB)-CpG marker with a high level of true positive in comparison to false positive results. Similar result was obtained using

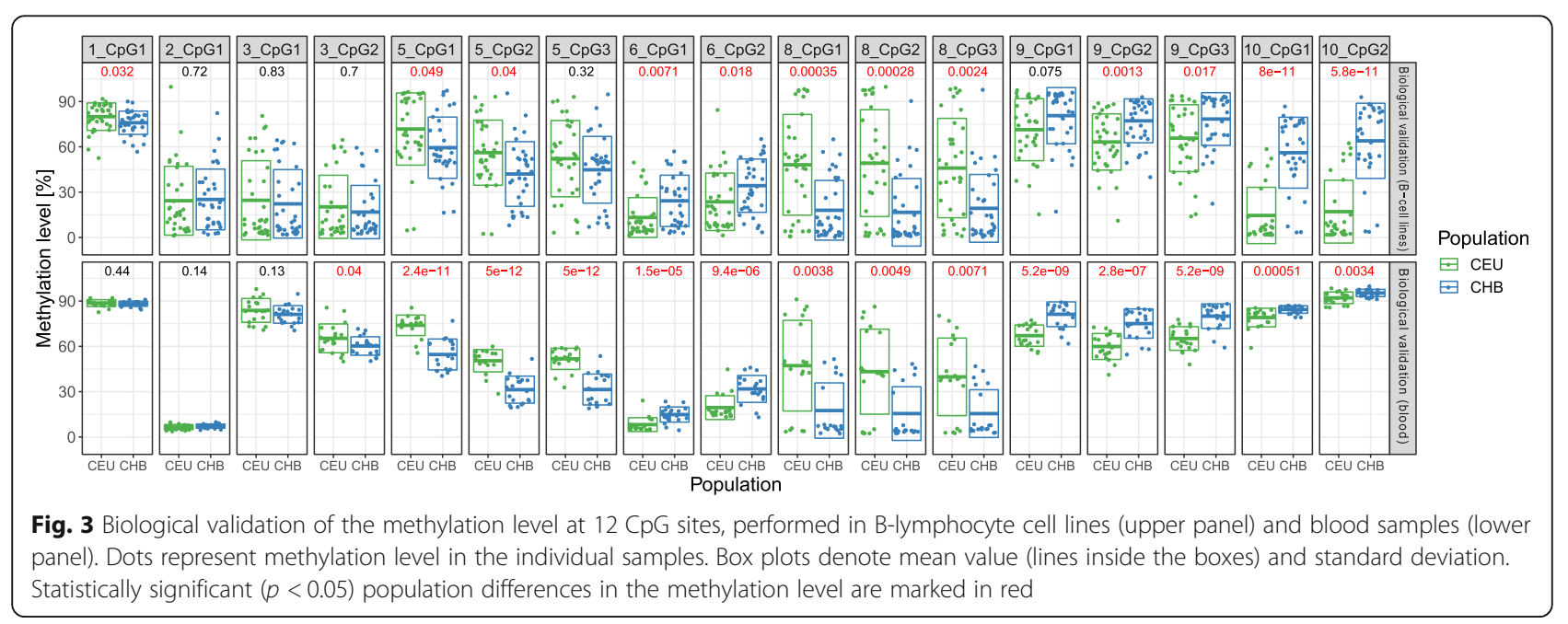




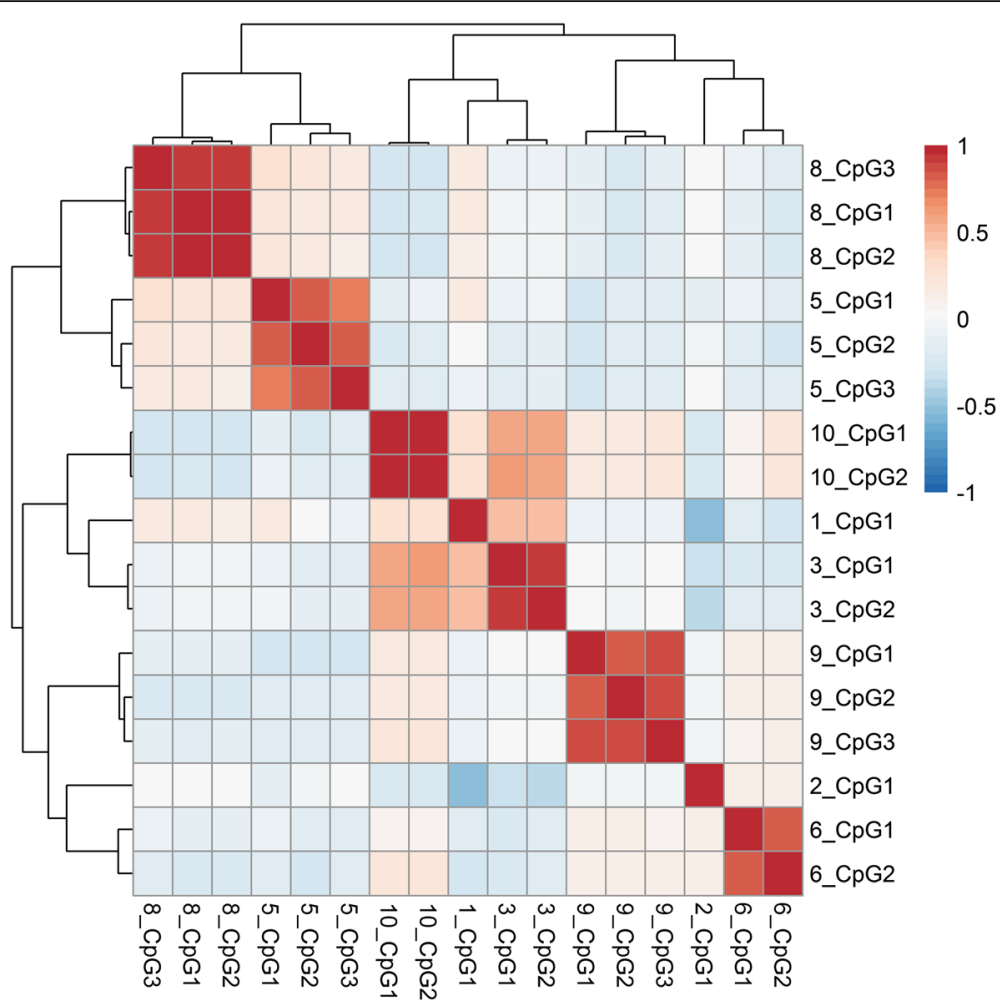

Fig. 4 Correlation matrix showing the results of Pearson correlation analysis. Analysis was performed using data from PyroAssays performed in 20 Blymphocyte cell lines ( $n=10$ from CEU, $n=10$ from CHB population). Pearson correlation coefficient values and directions are marked with different colors; positive correlation (from white to red on the color scale); negative correlation (from white to blue) (see color-bar next to the matrix)

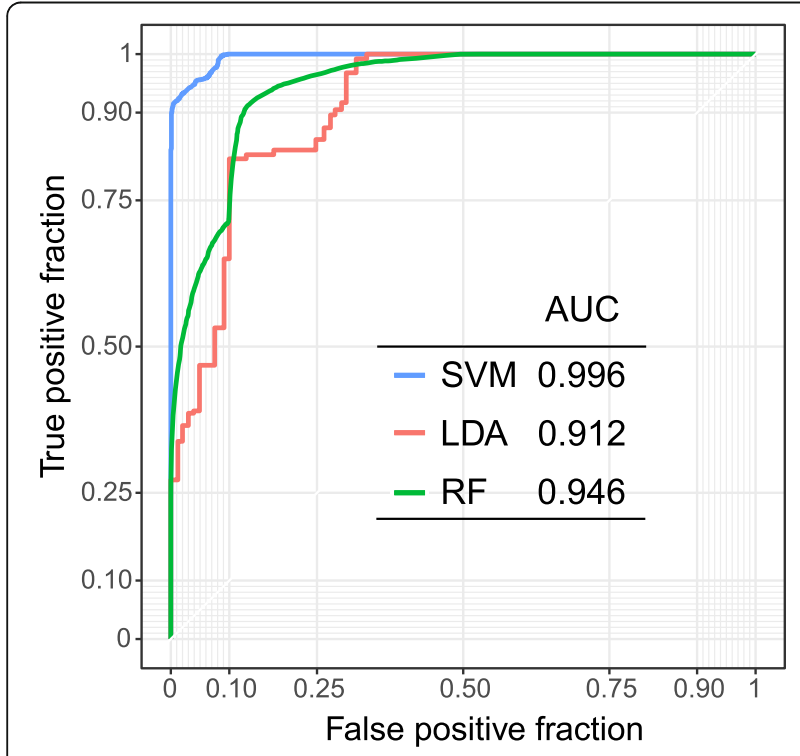

Fig. 5 Accuracy of the classification using three different classification methods. A ROC curve and AUC parameter were calculated for: support vector machines (SVM; blue line), linear discriminate analysis LDA (red line), and random forest (RF; green line). Results were obtained based on B-lymphocyte cell lines ( $n=20$ from CEU and CHB). The ROC curve was created by plotting the true positive fraction against the false positive fraction at various threshold settings all three tested classification methods (AUC $>0.9$ ), of which SVM was the most reliable (AUC $=0.996)$. The SVM validation performed on two independent datasets, B-lymphocyte cell lines $(n=48)$ and blood samples $(n=$ 40 ), showed a high accuracy of the classification power in both sets (> 85\%) (see Additional file 2).

Principle Component Analysis was used to assess the potential of the 8-site composite pop (CEU-CHB)-CpG marker to separate samples from two analyzed populations. While the vast majority of samples clustered according to their population affiliation, two populationspecific clusters were located in the close vicinity. The more accurate separation was obtained for blood samples (population-specific clusters were more separated from each other compared to B-cell samples) (Fig. 6a, b).

The variance distribution was attributed to the first $(\sim 30 \%)$ and the second $(\sim 17 \%)$ dimension in both Blymphocyte cell lines and blood samples. In both $\mathrm{PC}$ plots, markers 2_CpG1 (chr1:37939320, 6_CpG2 (chr15:45458818), 9_CpG3 (chr6:7051504) and 10_ CpG1 (chr1:36489272) correlated with each other and showed higher methylation level in CHB population, whereas markers 1_CpG1 (chr8:11418058), 3_CpG2 (chr3:178984959), 8_CpG1 (chr6:139013142) and 5_ CpG1 (chr5:132113734) showed higher metylation 

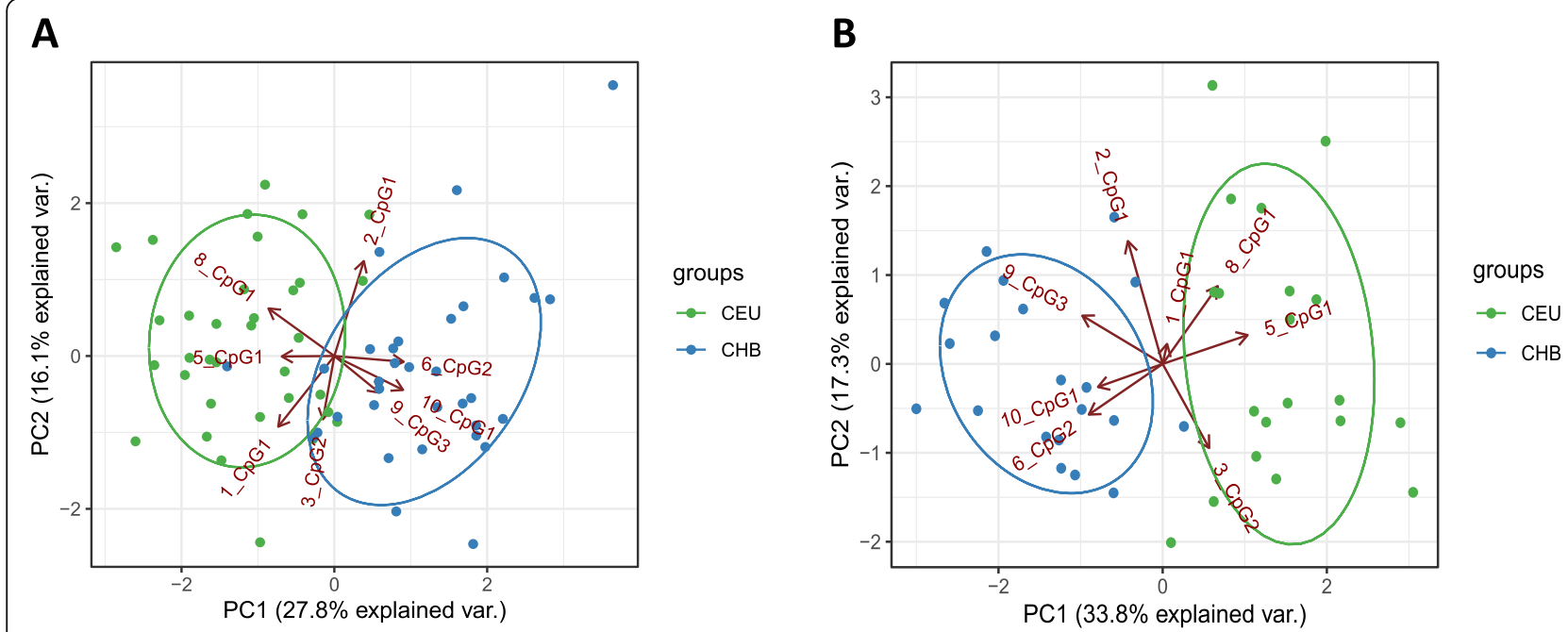

Fig. 6 PC analysis separating samples from two populations. Analysis was performed on B-lymphocyte cell lines ( $n=48$ from CEU and CHB; a and blood samples ( $n=40$ from CEU and CHB; $\mathbf{b}$. Vectors length denotes the power of influence of each feature on principal component, while vectors location show how variables correlate with one another. Dots represent individuals from CEU (green points) and CHB (blue points) population

level in CEU population. The weight of an individual CpG marker on the principle component was diverse, as indicated by the vectors length. What is interesting, most $\mathrm{CpG}$ markers had similar weight in PC analyzed in B-lymphocyte cell lines (Fig. 6a), while in blood sample, the impact of one marker, 1_CpG1 (chr8:11418058), was distinctly smaller (Fig. 6b).

An additional test was performed to assess the minimal number of popCpGs that would classify individuals of European and Chinese ancestry with high accuracy. The minimal number of seven unlinked pop-CpGs (10_CpG1 chr1:36489272, 6_CpG2 chr15:45458818, 1_CpG1 chr8: 11418058, 2_CpG1 chr1:37939320, 9_CpG3 chr6:7051504, 8_CpG1 chr6:139013142, 3_CpG2 chr3:178984959) had a high classification accuracy (AUC $\sim 1$, and precision $>0.8$ ) (Fig. 7, lower panel) in both B-lymphocyte cell lines and blood samples; discrimination potential obtained in peripheral blood samples (precision $=0.925$ ) was higher in comparison to B-lymphocyte cell lines (precision $=0.854$ ). In order to obtain similar discrimination power in both Blymphocyte cell lines and peripheral blood samples, we decided to retain the 8-site composite pop (CEU-CHB)-CpG marker to be used for methylation-based classification of $\mathrm{CEU}$ and $\mathrm{CHB}$ populations (see Fig. 7, lower panel).

To assess the population-discriminating potential of the 8-site composite pop (CEU-CHB)-CpG marker on the individuals of both genders, an in silico analysis was performed using additional DNA methylation data for Blymphocyte cell lines investigated on Illumina Infinium Human Methylation 450 BeadChip Array platform, obtained from GEO database (GSE36369). The SVM validation performed on two independent datasets: 93 Males
$(\mathrm{CEU}=47 ; \mathrm{CHB}=46)$ and 99 Females $(\mathrm{CEU}=49 ; \mathrm{CHB}=$ 50 ), showed a high accuracy of the classification power in both genders (> 89\%) (see Additional file 3).

Furthermore, a biological validation of the 8-point composite pop (CEU-CHB)-CpG marker was performed. Male and Female blood samples from CEU $(n=96)$ and CHB $(n=96)$ population were obtained from the same Illumina microarray experiment as before (GSE36369). Results, similar to those coming from B-lymphocyte cell lines, indicated high population discrimination potential of our 8-point marker, regardless of the gender (see Additional file 4).

\section{Discussion}

The aim of our study was to identify a set of CpG sites characterized by a significant difference in the DNA methylation profile between individuals of European and Chinese ancestry. Mainly adult males were analyzed. Analysis of $18 \mathrm{CEU}$ and $18 \mathrm{CHB}$ B-lymphocyte cell lines, performed on HM450K array, which measures the methylation of approximately 480,000 CpG sites across the human genome in parallel, revealed only $14 \mathrm{CpG}$ sites with significantly different methylation levels in the studied populations $\left(\mid \mathrm{M}_{\mathrm{av}-}\right.$ diff $\mid \geq 1.0$ and $\left.\mathrm{q}<0.05\right)$. According to the literature [41], $\mid \mathrm{M}_{\mathrm{av}-}$ diff $\mid \geq 1.0$ corresponds to a $20 \%$ difference in the methylation value. Such a small number of potentially populationdifferentiating $\mathrm{CpG}$ sites, with a relatively low interpopulation differences in the methylation level $\left(\left|\mathrm{M}_{\text {diff }}\right|\right.$ in the range of 1.1-2.9), stands in line with results from other studies. It has been estimated that only a small 

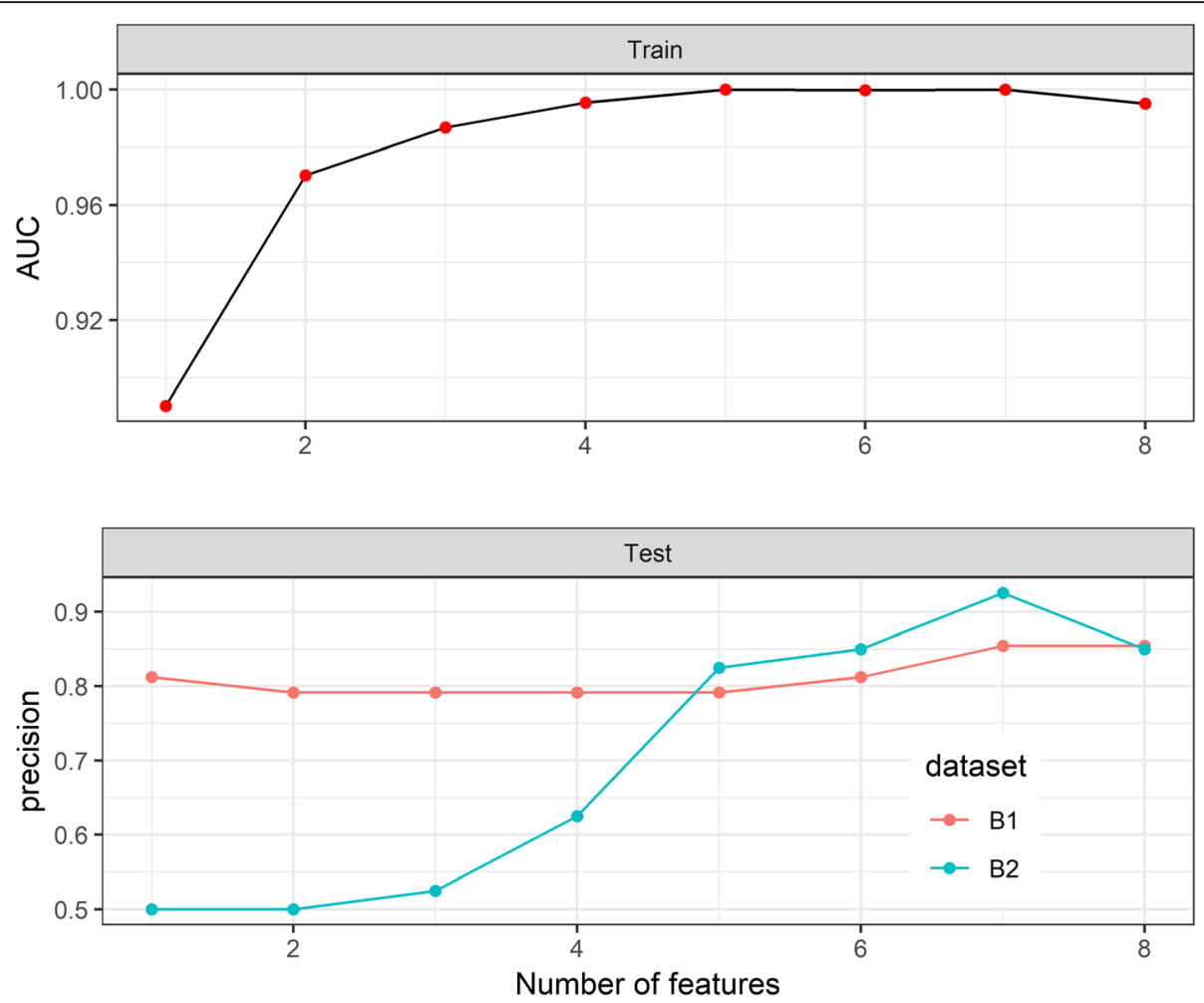

Fig. 7 Relation between the number of $\mathrm{CpG}$ used in a training group and the quality of classification. Upper panel: The value of AUC parameter obtained in the training group (B-cell lines: $\mathrm{CEU}, n=10 ; \mathrm{CHB}, n=10$ ) depending on the number of $\mathrm{CpG}$ studied. Lower panel: Precision of sample classification depending on the number of features (CpGs) analyzed in two tested groups: B-lymphocyte cell lines $(B 1$, red color, $n=48)$ and blood samples (B2, blue color, $n=40)$

fraction of CpGs across the genome stably varies in their methylation among human populations [18, 20, 42, 43].

A subset of 10 CpGs preselected in HM450K array experiment (cg24861686 chr8:11418058, cg03140118 chr1: 37939320, cg00862290 chr3:178984973, cg07904028 chr4: 6328508, cg08979191 chr5:132113734, cg04036182 chr15: 45458818, cg26367031 chr3:178984747, cg18136963 chr6: 139013146, cg07207043 chr6:7051497, cg23669876 chr1: 36489276), referred to as candidate pop-CpGs, with the highest inter-population differences in the mean methylation value $\left(\mid \mathrm{M}_{\mathrm{av} \_}\right.$diff $\mid \geq 1.2$ and $\left.\mathrm{q}-\mathrm{val}<0.05\right)$, was selected for further validation. Validation was done using pyrosequencing technique, regarded as a more sensitive method and widely used in DNA methylation studies [44, 45]. PyroAssays covered 10 candidate pop-CpGs, and several closely located neighboring CpGs, such that overall 20 CpG sites were tested.

Two-step validation was performed to exclude technical obstacles that could provide faulty results (technical validation in B-lymphocyte cell lines). In the next step, a biological validation in peripheral blood samples was performed to exclude the possibility that the interpopulation differences in the methylation level reflected specific conditions related to the maintenance of the $\mathrm{CHB}$ and CEU cell lines. Following these two steps. Six out of the eight PyroAssays tested on primary material displayed statistically significant inter-population differences in the methylation level $(p<0.05)$.

These results indicated that the candidate populationdifferentiating $\mathrm{CpG}$ sites selected based on the analysis of B-lymphocyte cell lines, after a proper validation, may be used as population-differentiating markers also in the primary cells (blood samples) (see also [19]).

Our results are especially interesting in the context of a widely discussed suitability of B-lymphocyte cell lines (lymphoblastoid cell lines, LCLs) for population studies on methylation [46, 47]. LCLs are a commonly used source of biological material due to their easy availability (Coriell repository resources), tissue homogeneity (exclusively B-lymphocytes) and known population origin. However, some recent studies revealed that laboratory treatment of LCLs, e.g. EBV transformation or specific conditions during culturing (e.g. repeated freeze-thaw cycles), may induce random DNA methylation alterations and thus produce misleading methylation results [46-48]. In this context, a comparison of raw methylation readouts collected from B-lymphocyte cell lines and from blood samples in our study revealed interesting observations. A high consistency in the methylation level was observed among CpGs examined within each 
PyroAssay, both in B-lymphocyte cell lines and in blood samples. However, the mean values of inter-population differences in blood samples were smaller than in Blymphocyte cell lines, and did not exceed $30 \%$ as opposed to nearly $50 \%$ in the cell lines. On the other hand, for the majority of $\mathrm{CpGs}$, the readouts representing methylation in individual cell lines were scattered, while those representing individual blood samples remained "tightly" clustered around the mean (except for PyroAssay 8 , see discussion below). The scattered methylation readouts observed in the cell lines could reflect the lack of homogeneity of technical (cell line maintenance etc.) and/or biological factors (age, and/or lifestyle of cell lines donors). Analysis of the reported age of Blymphocyte cell lines donors (wherever available) revealed no correlation with the methylation results. Aspects related to the cell line maintenance were beyond our control (cells were purchased from Coriell Repository), but these lines have been used in many studies and to our knowledge no systemic population differences have been reported. The small variance of readouts observed in the primary biological material is more surprising. Knowing that blood is a mixture of different cell types, and that blood donors were not controlled for their lifestyle (e.g. diet, smoking etc.), methylation readouts were expected to be more scattered. On the other hand, the number of blood samples used in the analysis was lower than that of the cell lines, and it is possible that increasing the size of tested group would affect the picture.

The only exception from the generally small variance of the methylation readouts in blood samples was PyroAssay 8 , where the distribution of readouts followed a characteristic tri-modal pattern. This pattern, when observed in HM450K array, has been described to reflect the presence of SNP in the examined CpG sites or in sequences targeted by Illumina probes (see $[40,49])$. Although all PyroAssays in this study were designed to avoid SNP-related bias, a tri-modal pattern observed in PyroAssay 8 prompted us to subject it to a careful scrutiny, to exclude the possible impact of the genomic sequences. Both in sillico analysis, performed in Genome Browser Database, and Sanger sequencing of several Blymphocyte cell lines and blood samples, did not reveal any SNPs/indels in either interrogated $\mathrm{CpG}$ sites and under the primers used in PyroAssays (data not shown). It is probable that, here also, increasing the number of samples could change this picture. In fact, an indication of a tri-modal distribution in PyroAssay 8 was also detectable in B-lymphocyte cell lines, but the larger number of samples blurred it into a cloudlike pattern (see Fig. 3).

To confirm the discriminating power of the composite pop (CEU-CHB)-CpG marker, composed of the validated pop-CpGs, a number of statistical analyzes were performed. All three algorithms (SVM, RF and LDA) used to test the sensitivity and specificity (ROC and AUC parameters) of population classification worked well in both types of the biological material (B-lymphocyte cell lines and blood samples), revealing high precision (>90\%) of sample population classification. What is more, our 8-point composite marker had a high population discrimination potential regardless of the gender, as shown by an in silico analysis of B-lymphocyte cell lines and blood samples.

According to the literature, a subset of populationspecific methylation markers ( $<500$ pop-CpG sites) allows to carry out discrimination of main human populations. The set of eight pop-CpGs described in our study is, to our knowledge, the smallest methylation-based composite marker able to discriminate two human populations [13, 20, 43]. Principal component analysis using the 8-site composite pop (CEU-CHB)-CpG marker clearly separated European and Chinese samples with respect to their population affiliation. What is interesting, a better classification was obtained in peripheral blood samples than in LCL material (see Fig. 6).

To better characterize our composite pop (CEUCHB)-CpG marker, we analyzed the genomic location of the differentially methylated CpGs. The vast majority of CpGs targeted by PyroAssays in this study were located outside of the, presumably evolutionary-conserved, $\mathrm{CpG}$ islands (see Table 1). CpG sites targeted by PyroAssays 5 and 8 were located in the shore regions $(\sim 2 \mathrm{~kb}$ from CpG islands, as defined by Illumina) of Septin8 and FLJ49/FLJ46906 genes, respectively. CpG sites targeted by PyroAssay 10 were situated in the body of AGO3 gene. The only sites located in $\mathrm{CpG}$ islands (of $S H F$ and RREB1 genes, respectively) were those targeted by PyroAssays 6 and 9. Our results therefore concord with other studies, which have indicated that inter-population differences in DNA methylation level are enriched outside CpG islands and are concentrated in regions flanking the islands (shores, shelfs) or in gene body regions [42, 43].

Genes, in which our pop-CpGs reside, are involved in various biological processes: apoptosis regulation $(S H F)$, expression regulation (FLJ49/FLJ46906), RNA interference (AGO3); or participate in distinct biological functions: transcription factor (RRB1), nucleotide binding protein (SEPT8). The biological relevance of the level of individual CpG sites methylation is still disputable [50]. However, it has been postulated that $\mathrm{CpG}$ sites located adjacent to functional genomics areas (CpG islands and/or shores) and representing similar methylation pattern due to potential effect on the chromatin structure, may play an important biological role [21]. In search for a putative long-range co-methylation, we examined five of the studied CpGs (cg08979191 chr5:132113734, cg04036182 chr15:45458818, cg18136963 chr6:139013146, cg07207043 
chr6:7051497, cg23669876 chr1: 36489276). Methylation status of the neighboring CpG sites, located $200 \mathrm{bp}$ upand downstream from the pop-CpGs (co-methylation), was examined in samples from both populations (results in Additional file 5), using data from our HM450K array study ([40], data accessible through GEO Series accession number: GSE73901). Four of five pop-CpGs, cg08979191 (chr5:132113734), cg04036182 (chr15: 45458818), cg18136963 (chr6:139013146), cg07207043 (chr6:7051497), had other Illumina-targeted CpGs in their vicinity (see Additional file 5). CpG sites located as far as $200 \mathrm{bp}$ down- or upstream of two of the "core" CpGs (cg08979191, chr5:132113734 and cg18136963, chr6:139013146), displayed statistically significant inter-population differences in the level of methylation $\left(\mathrm{IM}_{\mathrm{av}-}\right.$ diffI in the range 0.8-2.5) (for details see Additional file 5). Importantly, the "direction" of these differences was the same as in the "core" cg08979191 and cg18136963 (the reduced level of methylation in individuals of Chinese in comparison to European ancestry. All the co-methylated $\mathrm{CpG}$ sites were located in the shore regions flanking $\mathrm{CpG}$ islands, of SEPT8 and FLJ49/FLJ46906 genes, respectively. A highly correlated methylation level of CpG sites separated by $200 \mathrm{bp}$ suggests that cg08979191 (chr5:132113734) and cg18136963 (chr6:139013146) represent the methylation status of a longer region; this would be similar to the effect of linkage disequilibrium between SNPs in the human genome. However, it has to be kept in mind that Illumina HM450K array probes target a relatively small proportion of $\mathrm{CpG}$ sites in the human genome. A much larger number of neighboring $\mathrm{CpG}$ sites are present at the closer distance to these and the remaining pop-CpGs in our study; to examine methylation status of these sites, techniques addressing the whole genome should be employed, e.g. NGS technology.

DNA methylation constitutes an epigenetic switch in gene expression regulation $[19,20,36,51]$. The relation between gene promotor methylation status and transcriptional regulation is well known and widely studied (e.g. [20, 36, 52]. However, recent studies also indicated more complex relation among DNA methylation status of $\mathrm{CpG}$ located in gene body regions, and/or intragenic sites and gene expression [51, 53, 54]. To determine, whether differentially methylated CpGs in our study reflected population differences in gene expression status, we integrated DNA methylation and gene expression data obtained from our previous studies performed on the same set of B-lymphocyte cell lines and conducted on two micorarrays systems: HM450K array and HumanHT-12v4 Expression BeadChip Kit expression array. All candidate pop-CpGs listed in Table 1 were subjected to Pearson correlation analysis. The results clearly demonstrate that among 14 candidate pop-CpGs, only one cg24861686 (1_CpG1, chr8:11418058) located in the body of $B L K$ gene, showed positive correlation between the gene expression and the methylation status. Such a positive correlation observed between the methylation status of a CpG localized in the gene body and the gene expression, was also observed in other studies (e.g. $[36,51,55])$. Among four others CpG sites located in $B L K$ gene and tested in HM450K array, two: cg21701351 (chr8:11374774) and cg15685006 (chr8:11413044) were rejected from further analysis due to the presence of confounding features (SNPs/indels under probe and multi-site mapping). For two others: cg21497594, (chr8: 11366745) and cg21175976 (chr8:11421338), positioned in 5'UTR region and gene body, respectively, no statistically significant differences in the methylation level between study populations were identified $(q>0.05)$. Therefore, neither cg21497594 nor cg21175976 were subjected to Pearson correlation analysis.

These results are not surprising, since the regulation of gene expression is a complex process involving e.g. transcription factors, histone modification, non-coding RNA regulation [51, 56-58]. A straight methylation-expression correlation is rarely observed, or is noted exclusively in individual genes [51]. In conclusion, the biological meaning of the differential methylation status observed in the analyzed populations remains to be elucidated.

A relationship between the genome and the methylome, as well as an association of DNA methylation with the gene expression regulation, are frequently discussed in the literature. There is ample literature indicating that $2 / 3$ of methylation variability among population can be traced back to genetic ancestry $([15,20,36,37,43]$. Therefore, to investigate the relationship between our population- differentiating $\mathrm{CpG}$ sites and the genetic background, we performed an in silico analysis of the genetic variability in the region $\pm 10 \mathrm{~kb}$ around 14 selected pop-CpGs. For all tested pop-CpGs, the analysis showed the presence of a number of SNPs with Fst values in the range $0.00002-0.79$ in $20 \mathrm{~kb}$ region. Selected SNPs with the highest CEU-CHB Fst values (0.28-0.79) are shown in Additional file 6. Our analysis suggest that inter-population differences in the methylation level could be due to the genetic variability of the analyzed populations. However, to draw conclusions regarding correlations between our populationdifferentiating CpG sites and individual SNPs, detailed similar analysis is required with respect to using the genetic data of individual B-lymphocyte cell lines used in this project would be necessary.

\section{Conclusions}

Our results showed that even a small set of carefully selected differentially methylated CpGs (pop-CpGs), may 
be used to distinguish European and Chinese populations. Importantly, this composite pop (CEU-CHB)-CpG marker performs well in both lymphoblastoid cell lines and in non-homogenous blood samples regardless of a gender. The performance of our composite marker, estimated using different classification methods, was reasonably high for the limited number of examined samples, although this may change (either decrease or increase) when a larger number of sample are analyzed. Also, further studies using samples from other population groups need to be carried out.

The current knowledge regarding relation between epigenetics and environmental factors, as well as a transgeneration inheritance of methylation pattern (e.g. [36, 59]), is still limited. Nevertheless, it seems that discrimination between populations and inference of population origin of a sample, based on DNA methylation markers, is feasible and may add a new, additional dimension to medical and forensic casework, as earlier postulated $[1,60]$.

\section{Methods \\ DNA samples}

DNA samples from unrelated, healthy adult males and females representing European ancestry (mean age 38 years $\mathrm{SD} \pm 10.3$ years) and Chinese populations from Bejing with an exception of few samples from Japan (further referred to as $\mathrm{CEU}$ and $\mathrm{CHB}$, respectively) (for details see Additional file 7), were isolated either from commercially available B-lymphocyte cell lines (Coriell Cell Repositories) or from samples of peripheral blood (CEU $n=20, \mathrm{CHB} n=20$ ).

Both B-lymphocyte cell lines and peripheral blood samples used in this study underwent identical procedures including: DNA isolation (QIAamp DNA Blood Mini Kit, Qiagen), evaluation of its purity (Qubit, DSDNA H5 Assay Kit, Life Technology), and bisulfite treatment (EZ DNA Methylation-Gold Kit. Zymo Research). Five hundredng of purified DNA from Blymphocyte cell lines $(n=90)$, and peripheral blood $(n=$ 40) was converted with bisulfite solution using EZ DNA Methylation-GoldTM Kit (Zymo Research, Germany), according to the manufacturer's protocol.

\section{Study design}

The study consisted of four main phases: selection of candidate pop-CpGs, two-step validation, and statistical tests (Fig. 1).

\section{Selection of candidate pop-CpGs: identification of differentially metylated $C p G$ sites based on Human Methylation 450 BeadChip Array}

B-lymphocyte cell lines from CEU $(n=18)$ and CHB ( $n=18)$ were examined on Illumina Infinium HumanMethylation 450 BeadChip Array (further referred to as
HM450K array), according to the manufacturer-specified procedure. All analytical procedures, such as microarray technical quality evaluation, as well as statistical approach implemented in microarray data analysis, have been presented in detail in our previous publication see [40].

\section{Technical validation: pyrosequencing assay design and optimization}

Technical validation step was performed in a subset of B-lymphocyte cell lines previously analyzed by HM450K array. Pyrosequencing assays (further referred as PyroAssays) were designed to validate candidate pop-CpG sites preselected in HM450K array experiment for which effective PyroAssays could be designed (Assay score in PyroMark Assay Design Software $\geq 75$, no CpGs under PyroAssay primers); in some cases, PyroAssays covered additional CpGs located in the close proximity (less than 25 bp upstream or downstream) of the selected candidate pop-CpGs (see Table 2 in Result section).

Wherever possible, PyroAssays were designed to analyze CpGs on the same DNA strand as in the microarray experiment, to eliminate possible differences in the CpG methylation status depending on the DNA strands (the only exceptions were PyroAssays 2, 4, 7) (for details see Additional file 8).

PCR reaction conditions (PCR program and further sample workflow) are available in Additional file 8. Primers for PyroAssays were designed using PyroMark Assay Design Software 2.0.1.15 (Qiagen). Only those PyroAssays, for which specific PCR products were obtained for both bisulfite converted study samples and for methylated/unmethylated controls, were used in further analyses (see Additional file 1).

The quality of methylation results collected from pyrosequencing reactions was assessed based on a series of dilution curves obtained for all the PyroAssays (see Additional file 9).

\section{Biological validation: pyrosequencing assays in independent samples}

CpGs that passed technical validation were further tested in two steps. In the first one, PyroAssays were examined in an independent set of B-lymphocyte cell lines from both populations (CEU $n=35$; $\mathrm{CHB} n=35$ ); in the second step, PyroAssays were tested in peripheral blood samples (CEU $n=20$; CHB $n=20$ ) (see Fig. 1).

The same technical conditions (initial sample preparation, PCR reaction, Pyrosequencing process) were applied in both biological validation steps (see Additional files: 1 and 8).

\section{Statistical analysis}

Selection of the best non-redundant pop-CpGs from among those that passed technical and biological 
validation steps was conducted using beta regression test from the betareg Bioconductor package [61], with Benjamini Hochberg multiple testing correction.

The selected set of CpGs was then examined for its population-discriminating potential. Sample classification was conducted using three methods: support vector machines (SVM) with linear kernel, random forest (RF) and linear discriminant analysis (LDA). Prior to the classification process, correlated CpGs were removed; it was done by retaining only those with the lowest, adjusted $p$ values in the beta regression test for the technical validation dataset (20 samples), which was also used to select the best classification method. The predictive ability of the selected set of pop-CpGs was assessed using each of the classification methods, with 10-fold cross-validation, repeated 1000 times. In all cases classification was conducted using all possible combinations of 1 to $8 \mathrm{CpGs}$ identified as differentially methylated.

The best classification method in terms of AUC (area under ROC curve) was than validated using two independent datasets from $48 \mathrm{~B}$-lymphocyte cell lines and 40 total blood samples; all of the datasets were balanced (equal number of CEU and CHB samples). Classification was conducted in $\mathrm{R}$ with caret library and plotROC and ggplot2 used for visualization purposes.

Principal Component Analysys (PCA) was carried out in $\mathrm{R}$ using prcomp function from the stats package and visualized with the ggbiplot library.

In silico analysis of the genetic variability in the region $\pm 10 \mathrm{~kb}$ around 14 selected pop-CpGs was carried out in $\mathrm{R}$ using Pegas package. Genomic data for a representative group of samples from both study populations $(n=$ $198 \mathrm{CEU}$ and $n=206 \mathrm{CHB}$ ) was obtained from $1000 \mathrm{Ge}-$ nomes database.

\section{Supplementary information}

Supplementary information accompanies this paper at https://doi.org/10. 1186/s12864-020-07092-x.

Additional file 1. Pyrosequencing procedures

Additional file 2. A results of 3 classifiers cross-validation.

Additional file 3. A results of SVM classification performed on Male and Female B-lymphocyte cell lines obtained from GEO database (GSE36369).

Additional file 4. Biological validation of 8-point composite pop (CEU(HB)-diff-met marker performed in blood samples.

Additional file 5. Comethylation results of Pyrosequencing Assays that underwent biological validation.

Additional file 6. A list of pop-CpGs and selected SNPs showing the highest CEU-CHB Fst value.

Additional file 7. A list of B-cell lines used in Illumina Microarray analysis and Pyrosequencing study.

Additional file 8. Pyrosequencig Assay designing and reaction optimization.

Additional file 9. Evaluation of PyroAssays sensitivity.

\section{Abbreviations}

CEU: Utah residents (CEPH) with Northern and Western European ancestry; CHB: Han Chinese in Beijing, China; HM450K array: Illumina Infinium Human Methylation 450 BeadChip Array; pop-CpGs: A set of CpG sites differentiating European and Chinese population based on methylation level differences; PyroAssays: Pyrosequencing assays

\section{Acknowledgments \\ We thank Prof. Giefing, Prof. Słomski, and Prof. Szalata for offering excellent training in pyrosequencing technology, opportunity to use their \\ Pyrosequencers and for valuable scientific discussions.}

\section{Authors' contributions}

PDR participated in the design of the study, pyrosequencing validation, data analysis, and drafted manuscript; RJ performed statistical analysis, JP participated in pyrosequencing assays designing, data analysis and offered technical training in pyrosequencing technology, MW and EZ participated in the design of the study and critically revised the manuscript. All authors have read and approved the final manuscript.

\section{Funding}

The study was supported by the grant AriaDNA OR00 002712 from The National Centre for Research and Development and MINIATURA (2017 101/ XAIZ8|00096) from National Science Center Poland. The funding bodies played no role in the design of the study and collection, analysis, and interpretation of data and in writing the manuscript.

\section{Availability of data and materials}

The datasets generated and/or analyzed during the current study are available in GEO database with accession number GSE73901.

The genomic sequences surrounding the study pop-CpGs were obtained from USCS Genome Browser database (https://genome.ucsc.edu/) based on genomic CpG site location (see main text).

For an in silico analysis we used data from GEO database (GSE36369) and from 1000 Genome Project (https://www.internationalgenome.org).

All of the datasets supporting the results of this article are included within the article and its Additional files.

\section{Ethics approval and consent to participate}

The samples of peripheral blood were collected from anonymous healthy donors and were obtained with their informed consent. The donors were explicitly informed about the aim of the sample collection by providing them with a short description of the proposed project. The informed consent was taken in verbal form to ensure the anonymity of the donors, who did not wish to disclose their names. Such a procedure was approved by the Bioethical Committee at the Central Clinical Hospital of the Ministry of Interior in Warsaw, which gave the appropriate consent (No 67/2010). The B-lymphocyte lines used in the project were purchased from Corriell depository, and were selected to represent studied populations (European, CEU; and Chinese, CHB); catalogue numbers are listed in Additional file 7. No details allowing identification of the individuals were used in the manuscript (in particular, the column describing age was replaced by the age range, without reference to particular individuals).

Consent for publication

Not applicable.

Competing interests

The authors declare that they have no competing interests.

\section{Author details}

${ }^{1}$ Institute of Human Genetics, Polish Academy of Sciences, Strzeszynska 32, 60-479 Poznan, Poland. '2Silesian University of Technology, Akademicka 16, 44-100 Gliwice, Poland.

Received: 31 March 2020 Accepted: 22 September 2020

Published online: 12 October 2020

\section{References}

1. Kader F, Ghai M. DNA methylation and application in forensic sciences. Forensic Sci Int. 2015;249:255-65. 
2. Hernando-Herraez I, Garcia-Perez R, Sharp AJ, Marques-Bonet T. DNA methylation: insights into human evolution. PLoS Genet. 2015;11(12): e1005661.

3. Levenson W, Melnikov AA. DNA methylation as clinically useful biomarkerslight at the end of the tunnel. Pharmaceuticals (Basel). 2012;5(1):94-113.

4. Unnikrishnan A, Freeman WM, Jackson J, Wren JD, Porter H, Richardson A. The role of DNA methylation in epigenetics of aging. Pharmacol Ther. 2019; 195:172-85.

5. Spolnicka M, Pospiech E, Peplonska B, Zbiec-Piekarska R, Makowska Z, Pieta A, et al. DNA methylation in ELOVL2 and C1orf132 correctly predicted chronological age of individuals from three disease groups. Int J Legal Med. 2018;132(1):1-11.

6. Novembre J, Johnson T, Bryc K, Kutalik Z, Boyko AR, Auton A, et al. Genes mirror geography within Europe. Nature. 2008;456(7218):98-U5.

7. Li JZ, Absher DM, Tang H, Southwick AM, Casto AM, Ramachandran S, et al. Worldwide human relationships inferred from genome-wide patterns of variation. Science. 2008;319(5866):1100-4.

8. Price AL, Butler J, Patterson N, Capelli C, Pascali VL, Scarnicci F, et al. Discerning the ancestry of European Americans in genetic association studies. PLoS Genet. 2008;4(1):9-17.

9. Sudmant PH, Mallick S, Nelson BJ, Hormozdiari F, Krumm N, Huddleston J, et al. Global diversity, population stratification, and selection of human copy-number variation. Science. 2015;349(6253):aab3761.

10. Phillips C, Parson W, Lundsberg B, Santos C, Freire-Aradas A, Torres M, et al. Building a forensic ancestry panel from the ground up: the EUROFORGEN global AIM-SNP set. Forensic Sci Int Genet. 2014;11:13-25.

11. Daca-Roszak P, Pfeifer A, Zebracka-Gala J, Jarzab B, Witt M, Zietkiewicz E. EurEAs_Gplex-a new SNaPshot assay for continental population discrimination and gender identification. Forensic Sci Int Genet. 2016;20:89100.

12. Brandstatter A, Salas A, Niederstatter H, Gassner C, Carracedo A, Parson W. Dissection of mitochondrial superhaplogroup $\mathrm{H}$ using coding region SNPS. Electrophoresis. 2006;27(13):2541-50.

13. Husquin LT, Rotival M, Fagny M, Quach $H$, Zidane $N$, McEwen LM, et al. Exploring the genetic basis of human population differences in DNA methylation and their causal impact on immune gene regulation. Genome Biol. 2018;19(1):222.

14. Lam LL, Emberly E, Fraser HB, Neumann SM, Chen E, Miller GE, et al. Factors underlying variable DNA methylation in a human community cohort. Proc Natl Acad Sci U S A. 2012;109(Suppl 2):17253-60.

15. Adkins RM, Krushkal J, Tylavsky FA, Thomas F. Racial differences in genespecific DNA methylation levels are present at birth. Birth Defects Res A Clin Mol Teratol. 2011;91(8):728-36

16. Terry MB, Ferris JS, Pilsner R, Flom JD, Tehranifar $P$, Santella RM, et al. Genomic DNA methylation among women in a multiethnic New York City birth cohort. Cancer Epidemiol Biomark Prev. 2008;17(9):2306-10.

17. Zhang FF, Cardarelli R, Carroll J, Fulda KG, Kaur M, Gonzalez K, et al. Significant differences in global genomic DNA methylation by gender and race/ethnicity in peripheral blood. Epigenetics. 2011;6(5):623-9.

18. Song MA, Brasky TM, Marian C, Weng DY, Taslim C, Dumitrescu RG, et al. Racial differences in genome-wide methylation profiling and gene expression in breast tissues from healthy women. Epigenetics. 2015;10(12): 1177-87.

19. Fraser HB, Lam LL, Neumann SM, Kobor MS. Population-specificity of human DNA methylation. Genome Biol. 2012:13(2):R8.

20. Heyn H, Moran S, Hernando-Herraez I, Sayols S, Gomez A, Sandoval J, et al. DNA methylation contributes to natural human variation. Genome Res. 2013;23(9):1363-72.

21. Giuliani C, Sazzini M, Bacalini MG, Pirazzini C, Marasco E, Fontanesi E, et al. Epigenetic variability across human populations: a focus on DNA methylation profiles of the KRTCAP3, MAD1L1 and BRSK2 genes. Genome Biol Evol. 2016;8(9):2760-73.

22. Zhao LN, Liu D, Xu J, Wang ZY, Chen Y, Lei CG, et al. The framework for population epigenetic study. Brief Bioinform. 2018;19(1):89-100.

23. Daca-Roszak P, Swierniak M, Jaksik R, Tyszkiewicz T, Oczko-Wojciechowska M, Zebracka-Gala J, et al. Transcriptomic population markers for human population discrimination. BMC Genet. 2018;19:1471-2156.

24. King K, Murphy S, Hoyo C. Epigenetic regulation of Newborns' imprinted genes related to gestational growth: patterning by parental race/ethnicity and maternal socioeconomic status. J Epidemiol Community Health. 2015; 69(7):639-47.
25. Mozhui K, Smith AK, Tylavsky FA. Ancestry Dependent DNA Methylation and Influence of Maternal Nutrition. Plos One. 2015;10(3):e0118466.

26. Plusquin M, Guida F, Polidoro S, Vermeulen R, Raaschou-Nielsen O, Campanella G, et al. DNA methylation and exposure to ambient air pollution in two prospective cohorts. Environ Int. 2017;108:127-36.

27. Bollati V, Baccarelli A. Environmental epigenetics. Heredity (Edinb). 2010; 105(1):105-12

28. van Dongen J, Nivard MG, Willemsen G, Hottenga JJ, Helmer Q, Dolan CV, et al. Genetic and environmental influences interact with age and sex in shaping the human methylome. Nat Commun. 2016;7:11115.

29. Rowlatt A, Hernández-Suárez G, Sanabria-Salas MC, Serrano-López M, Rawlik K, Hernandez-Illan E, et al. The heritability and patterns of DNA methylation in normal human colorectum. Hum Mol Genet. 2016;25(12):2600-11.

30. Galanter JM, Gignoux CR, Oh SS, Torgerson D, Pino-Yanes M, Thakuri N, et al. Differential methylation between ethnic sub-groups reflects the effect of genetic ancestry and environmental exposures. Elife. 2017;6:24.

31. Smith ZD, Chan MM, Humm KC, Karnik R, Mekhoubad S, Regev A, et al. DNA methylation dynamics of the human preimplantation embryo. Nature. 2014:511(7511):611-5.

32. Rahmani E, Shenhav L, Schweiger R, Yousefi P, Huen K, Eskenazi B, et al. Genome-wide methylation data mirror ancestry information. Epigenetics Chromatin. 2017;10:1.

33. Kwabi-Addo B, Wang S, Chung W, Jelinek J, Patierno SR, Wang BD, et al. Identification of differentially methylated genes in normal prostate tissues from African American and Caucasian men. Clin Cancer Res. 2010;16(14):3539-47.

34. Liu J, Morgan M, Hutchison K, Calhoun VD. A study of the influence of sex on genome wide methylation. PLoS One. 2010;5(4):e10028.

35. Y-y X, Y-b D, X-q L, X-m C, Cheng S-q, L-b L, et al. Racial/ethnic disparities in human DNA methylation. Biochimica Et Biophysica Acta Rev Cancer. 2014; 1846(1):258-62.

36. Bell JT, Pai AA, Pickrell JK, Gaffney DJ, Pique-Regi R, Degner JF, et al. DNA methylation patterns associate with genetic and gene expression variation in HapMap cell lines. Genome Biol. 2011;12(1):R10.

37. Moen EL, Zhang X, Mu W, Delaney SM, Wing C, McQuade J, et al. Genomewide variation of cytosine modifications between European and African populations and the implications for complex traits. Genetics. 2013;194(4):987.

38. Winnefeld M, Brueckner B, Groenniger E, Staeb F, Wenck H, Lyko F. Stable ethnic variations in DNA methylation patterns of human skin. J Investig Dermatol. 2012;132(2):466-8.

39. Bormann F, Rodriguez-Paredes M, Hagemann S, Manchanda H, Kristof B Gutekunst J, et al. Reduced DNA methylation patterning and transcriptional connectivity define human skin aging. Aging Cell. 2016;15(3):563-71.

40. Daca-Roszak P, Pfeifer A, Zebracka-Gala J, Rusinek D, Szybinska A, Jarzab B, et al. Impact of SNPs on methylation readouts by Illumina Infinium HumanMethylation450 BeadChip Array: implications for comparative population studies. BMC Genomics. 2015;16:1003.

41. Marabita F, Almgren M, Lindholm ME, Ruhrmann S, Fagerstrom-Billai F, Jagodic $M$, et al. An evaluation of analysis pipelines for DNA methylation profiling using the Illumina HumanMethylation450 BeadChip platform. Epigenetics. 2013;8(3):333-46.

42. Byun H-M, Siegmund KD, Pan F, Weisenberger DJ, Kanel G, Laird PW, et al. Epigenetic profiling of somatic tissues from human autopsy specimens identifies tissue- and individual-specific DNA methylation patterns. Hum Mol Genet. 2009;18(24):4808-17.

43. Carja O, Maclsaac JL, Mah SM, Henn BM, Kobor MS, Feldman MW, et al. Worldwide patterns of human epigenetic variation. Nat Ecol Evol. 2017; 1(10):1577-83

44. Potapova A, Albat C, Hasemeier B, Haeussler K, Lamprecht S, Suerbaum S, et al. Systematic cross-validation of 454 sequencing and pyrosequencing for the exact quantification of DNA methylation patterns with single CpG resolution. BMC Biotechnol. 2011;11:6.

45. Brakensiek K, Wingen LU, Langer F, Kreipe H, Lehmann U. Quantitative highresolution $\mathrm{CpG}$ island mapping with pyrosequencing reveals diseasespecific methylation patterns of the CDKN2B gene in myelodysplastic syndrome and myeloid leukemia. Clin Chem. 2007;53(1):17-23.

46. Grafodatskaya D, Choufani S, Ferreira JC, Butcher DT, Lou Y, Zhao C, et al. EBV transformation and cell culturing destabilizes DNA methylation in human lymphoblastoid cell lines. Genomics. 2010;95(2):73-83.

47. Sun YV, Turner ST, Smith JA, Hammond PI, Lazarus A, Van De Rostyne JL, et al. Comparison of the DNA methylation profiles of human peripheral blood cells and transformed B-lymphocytes. Hum Genet. 2010;127(6):651-8. 
48. Caliskan M, Cusanovich DA, Ober C, Gilad Y. The effects of EBV transformation on gene expression levels and methylation profiles. Hum Mol Genet. 2011;20(8):1643-52.

49. Naeem H, Wong NC, Chatterton Z, Hong MKH, Pedersen JS, Corcoran NM, et al. Reducing the risk of false discovery enabling identification of biologically significant genome-wide methylation status using the HumanMethylation450 array. BMC Genomics. 2014;15:51.

50. Wessely F, Emes RD. Identification of DNA methylation biomarkers from Infinium arrays. Front Genet. 2012;3:161.

51. Zhong H, Kim S, Zhi D, Cui X. Predicting gene expression using DNA methylation in three human populations. PeerJ. 2019;7:e6757.

52. Portela A, Esteller M. Epigenetic modifications and human disease. Nat Biotechnol. 2010;28(10):1057-68.

53. Grundberg E, Meduri E, Sandling JK, Hedman AK, Keildson S, Buil A, et al. Global analysis of DNA methylation variation in adipose tissue from twins reveals links to disease-associated variants in distal regulatory elements. Am J Hum Genet. 2013;93(5):876-90.

54. Wagner JR, Busche S, Ge B, Kwan T, Pastinen T, Blanchette M. The relationship between DNA methylation, genetic and expression interindividual variation in untransformed human fibroblasts. Genome Biol. 2014; 15(2):R37.

55. Jones PA. Functions of DNA methylation: islands, start sites, gene bodies and beyond. Nat Rev Genet. 2012;13(7):484-92

56. Verdin $\mathrm{E}, \mathrm{Ott} \mathrm{M} .50$ years of protein acetylation: from gene regulation to epigenetics, metabolism and beyond. Nat Rev Mol Cell Biol. 2015;16. England:258-64.

57. Janowski BA, Huffman KE, Schwartz JC, Ram R, Hardy D, Shames DS, et al. Inhibiting gene expression at transcription start sites in chromosomal DNA with antigene RNAs. Nat Chem Biol. 2005; 1(4):216-22.

58. Kaikkonen MU, Lam MT, Glass CK. Non-coding RNAs as regulators of gene expression and epigenetics. Cardiovasc Res. 2011;90(3):430-40.

59. Heard E, Martienssen RA. Transgenerational epigenetic inheritance: myths and mechanisms. Cell. 2014;157(1):95-109.

60. Vidaki A, Kayser M. Recent progress, methods and perspectives in forensic epigenetics. Forensic Sci Int Genet. 2018:37:180-95.

61. Francisco C-N. Beta Regression in R. In: Zeileis A, editor. Journal of Statistical Software; 2010. p. 1-24.

\section{Publisher's Note}

Springer Nature remains neutral with regard to jurisdictional claims in published maps and institutional affiliations.

Ready to submit your research? Choose BMC and benefit from:

- fast, convenient online submission

- thorough peer review by experienced researchers in your field

- rapid publication on acceptance

- support for research data, including large and complex data types

- gold Open Access which fosters wider collaboration and increased citations

- maximum visibility for your research: over $100 \mathrm{M}$ website views per year

At $\mathrm{BMC}$, research is always in progress.

Learn more biomedcentral.com/submissions 